BULL. AUSTRAL. MATH. SOC.

VOL. $10(1974), 365-370$.

\title{
On common fixed points of mappings
}

\section{Kiyoshi Iséki}

The object of this paper is to study common fixed points of mappings of a complete metric space into itself. The results obtained are generalizations of Ray Theorems.

Recently Ray [2] and Wong [4] proved some interesting theorems about common fixed points of mappings of a complete metric space in itself. In this note, we shall prove some theorems about common fixed points which are generalizations of results in Ray [2].

THEOREM 1. Let $X$ be a complete metric space, $T_{n}(n=1,2, \ldots)$ a sequence of mappings of $X$ into itself. Suppose that there are nonnegative numbers $\alpha, \beta, \gamma$ such that for $x, y \in X$, $\rho\left(T_{i}(x), T_{j}(y)\right) \leq \alpha\left(\rho\left(x, T_{i}(x)\right)+\rho\left(y, T_{j}(y)\right)\right)$

$$
+\beta\left(\rho\left(x, T_{j}(y)\right)+\rho\left(y, T_{i}(x)\right)\right)+\gamma \rho(x, y),
$$

where $2 \alpha+2 \beta+\gamma<1$. Then the sequence of moppings $\left\{T_{n}\right\}$ has a unique corrmon fixed point.

Proof. Let $x_{0} \in X$. Put

$$
x_{n}=T_{n}\left(x_{n-1}\right), \quad(n=1,2, \ldots) ;
$$

then we have 


$$
\begin{aligned}
\rho\left(x_{1}, x_{2}\right) & =\rho\left(T_{1}\left(x_{0}\right), T_{2}\left(x_{1}\right)\right) \\
& \leq \alpha\left(\rho\left(x_{0}, x_{1}\right)+\rho\left(x_{1}, x_{2}\right)\right)+\beta\left(\rho\left(x_{0}, x_{2}\right)+\rho\left(x_{1}, x_{1}\right)\right)+\gamma \rho\left(x_{0}, x_{1}\right) \\
& =(\alpha+\gamma) \rho\left(x_{0}, x_{1}\right)+\alpha \rho\left(x_{1}, x_{2}\right)+\beta \rho\left(x_{0}, x_{2}\right) \\
& \leq(\alpha+\gamma) \rho\left(x_{0}, x_{1}\right)+\alpha \rho\left(x_{1}, x_{2}\right)+\beta\left(\rho\left(x_{0}, x_{1}\right)+\rho\left(x_{1}, x_{2}\right)\right) .
\end{aligned}
$$

Hence

$$
\rho\left(x_{1}, x_{2}\right) \leq \frac{\alpha+\beta+\gamma}{1-\alpha-\beta} \rho\left(x_{0}, x_{1}\right) .
$$

Similarly we have

$$
\begin{aligned}
\rho\left(x_{2}, x_{3}\right) & =\rho\left(T\left(x_{1}\right), T\left(x_{2}\right)\right) \\
& \leq(\alpha+\gamma) \rho\left(x_{1}, x_{2}\right)+\alpha \rho\left(x_{2}, x_{3}\right)+\beta\left(\rho\left(x_{1}, x_{2}\right)+\rho\left(x_{2}, x_{3}\right)\right) .
\end{aligned}
$$

Therefore, we have

$$
\rho\left(x_{2}, x_{3}\right) \leq \frac{\alpha+\beta+\gamma}{1-\alpha-\beta} \rho\left(x_{1}, x_{2}\right) .
$$

In general, we have

$$
\rho\left(x_{n}, x_{n+1}\right) \leq\left(\frac{\alpha+\beta+\gamma}{1-\alpha-\beta}\right)^{n} \rho\left(x_{0}, x_{1}\right) .
$$

This means that the sequence $\left\{x_{n}\right\}$ is a Cauchy sequence. Hence, by the completeness of $X,\left\{x_{n}\right\}$ converges to some point $x$ in $X$. For the point $x$,

$$
\begin{aligned}
\rho\left(x, T_{n}(x)\right) \leq & \rho\left(x, x_{m+1}\right)+\rho\left(x_{m+1}, T_{n}(x)\right) \\
= & \rho\left(x, x_{m+1}\right)+\rho\left(T_{m+1}\left(x_{m}\right), T_{n}(x)\right) \\
\leq & \rho\left(x, x_{m+1}\right)+\alpha\left(\rho\left(x_{m}, T_{m+1}\left(x_{m}\right)\right)+\rho\left(x, T_{n}(x)\right)\right) \\
& \quad+\beta\left(\rho\left(x_{m}, T_{n}(x)\right)+\rho\left(x, T_{m+1}\left(x_{m}\right)\right)\right)+\gamma \rho\left(x_{m}, x\right) \\
= & \rho\left(x, x_{m+1}\right)+\alpha\left(\rho\left(x_{m}, x_{m+1}\right)+\rho\left(x, T_{n}(x)\right)\right) \\
& \quad+\beta\left(\rho\left(x_{m}, T_{n}(x)\right)+\rho\left(x, x_{m+1}\right)\right)+\gamma \rho\left(x_{m}, x\right) .
\end{aligned}
$$

Letting $m \rightarrow \infty$, then we have

$$
\rho\left(x, T_{n}(x)\right) \leq(\alpha+\beta) \rho\left(x, T_{n}(x)\right) .
$$

Therefore $\rho\left(x, T_{n}(x)\right)=0$; that is, the point $x$ is a common fixed 
point of all $T_{n}$.

To show that $x$ is a unique common fixed point of all $T_{n}$, we consider a point $y$ in $X$ such that $T_{n}(y)=y$ for every $n$. Then we have

$$
\begin{aligned}
\rho(x, y) & =\rho\left(T_{n}(x), T_{n}(y)\right) \\
\leq & \alpha\left(\rho\left(x, T_{n}(x)\right)+\rho\left(y, T_{n}(y)\right)\right)+\beta\left(\rho\left(x, T_{n}(y)\right)+\rho\left(y, T_{n}(x)\right)\right) \\
& \quad+\gamma \rho(x, y)=(2 \beta+\gamma) \rho(x, y) .
\end{aligned}
$$

Hence $\rho(x, y)=0$; that is, $x=y$. This completes the proof of Theorem 1 .

THEOREM 2. Let $\left\{T_{n}\right\}$ be a sequence of mappings of a complete metric space $X$ into itself. Let $x_{n}$ be a fixed point of $T_{n}(n=1,2, \ldots)$, and suppose that $T_{n}$ converges uniformly to $T_{0}$. If $T_{0}$ satisfies the condition

(I) $\rho\left(T_{0}(x), T_{0}(y)\right) \leq \alpha\left(\rho\left(x, T_{0}(x)\right)+\rho\left(y, T_{0}(y)\right)\right)$

$$
+\beta\left(\rho\left(x, T_{0}(y)\right)+\rho\left(y, T_{0}(x)\right)\right)+\gamma \rho(x, y),
$$

where $\alpha, \beta, \gamma$ are non-negative and $2 \alpha+2 \beta+\gamma<1$, then $\left\{x_{n}\right\}$ converges to the fixed point $x_{0}$ of $T_{0}$.

Under condition (1), $T_{0}$ has a unique fixed point by a result of Ćirić [1] (quoted from Rus [3], p. 21).

Proof. Let $\varepsilon>0$ be given; then there is a natural number $N$ such that

$$
\rho\left(T_{n}(x), T_{0}(x)\right)<\varepsilon
$$

for all $x \in X$ and $N \leq n$. Hence 
368

Kiyoshi Iséki

$$
\begin{aligned}
\rho\left(x_{n}, x_{0}\right)=\rho\left(T_{n}\left(x_{n}\right), T_{0}\left(x_{0}\right)\right) \\
\leq \rho\left(T_{n}\left(x_{n}\right), T_{0}\left(x_{n}\right)\right)+\rho\left(T_{0}\left(x_{n}\right), T_{0}\left(x_{0}\right)\right) \\
\leq \rho\left(T_{n}\left(x_{n}\right), T_{0}\left(x_{n}\right)\right)+\alpha\left(\rho\left(x_{n}, T_{0}\left(x_{n}\right)\right)+\rho\left(x_{0}, T_{0}\left(x_{0}\right)\right)\right) \\
\quad+\beta\left(\rho\left(x_{n}, T_{0}\left(x_{0}\right)\right)+\rho\left(x_{0}, T_{0}\left(x_{n}\right)\right)\right)+\gamma \rho\left(x_{n}, x_{0}\right) \\
\leq \rho\left(T_{n}\left(x_{n}\right), T_{0}\left(x_{n}\right)\right)+(\alpha+\beta) \rho\left(T_{n}\left(x_{n}\right), T_{0}\left(x_{n}\right)\right) \\
\quad+(\alpha+\beta)\left(\rho\left(x_{0}, x_{n}\right)+\rho\left(T_{n}\left(x_{n}\right), T_{0}\left(x_{n}\right)\right)\right)+\gamma \rho\left(x_{n}, x_{0}\right) \\
=(1+2(\alpha+\beta)) \rho\left(T_{n}\left(x_{n}\right), T_{0}\left(x_{n}\right)\right)+((\alpha+\beta)+\gamma) \rho\left(x_{n}, x_{0}\right) .
\end{aligned}
$$

Hence

$$
(1-(\alpha+\beta+\gamma)) \rho\left(x_{n}, x_{0}\right) \leq(1+2(\alpha+\beta)) \rho\left(T_{n}\left(x_{n}\right), T_{0}\left(x_{n}\right)\right) \text {. }
$$

From the hypotheses, $2(\alpha+\beta)+\gamma<1$. Hence, for $n \geq N$, we have

$$
\rho\left(x_{n}, x_{0}\right) \leq \frac{1+2(\alpha+\beta)}{1-(\alpha+\beta+\gamma)} \varepsilon,
$$

which shows that $\left\{x_{n}\right\}$ converges to $x_{0}$. We complete the proof.

THEOREM 3. Let $T_{n}(n=1,2, \ldots)$ be a sequence of mappings with fixed point $x_{n}$ of a metric space $X$ into itself. Suppose that

$$
\begin{aligned}
\rho\left(T_{n}(x), T_{n}(y)\right) \leq \alpha\left(\rho\left(x, T_{n}(x)\right)\right. & \left.+\rho\left(y, T_{n}(y)\right)\right) \\
& +\beta\left(\rho\left(x, T_{n}(y)\right)+\rho\left(y, T_{n}(x)\right)\right)+\gamma \rho(x, y),
\end{aligned}
$$

where $\alpha, \beta, \gamma$ are nonnegative and $2 \alpha+2 \beta+\gamma<1$. If $\left\{T_{n}\right\}$ converges to a mapping $T_{0}$, and $x_{0}$ is an accumulation point of $\left\{x_{n}\right\}$, then $x_{0}$ is a fixed point of $T_{0}$.

Proof. Since $x_{0}$ is an accumulation point of the set $\left\{x_{n}\right\}$, there is a subsequence $\left\{x_{n_{i}}\right\}$ of $\left\{x_{n}\right\}$ which converges to $x_{0}$ :

$$
\begin{aligned}
\rho\left(x_{0}, T_{0}\left(x_{0}\right)\right) \leq \rho\left(x_{0}, T_{n_{i}}\left(x_{n_{i}}\right)\right) & \\
& +\rho\left(T_{n_{i}}\left(x_{n_{i}}\right), T_{n_{i}}\left(x_{0}\right)\right)+\rho\left(T_{n_{i}}\left(x_{0}\right), T_{0}\left(x_{0}\right)\right) .
\end{aligned}
$$

Let $\varepsilon>0$; then there is a natural number $N$ such that

https://doi.org/10.1017/S0004972700041058 Published online by Cambridge University Press 
Common fixed points

369

$$
\begin{array}{r}
\rho\left(x_{0}, x_{n_{i}}\right)<\varepsilon, \\
\rho\left(T_{n_{i}}\left(x_{0}\right), T_{0}\left(x_{0}\right)\right)<\varepsilon,
\end{array}
$$

for $N \leq n_{i}$. Hence for $N \leq n_{i}$, we have

$$
\rho\left(x_{0}, T_{0}\left(x_{0}\right)\right)<2 \varepsilon+\rho\left(T_{n_{i}}\left(x_{n_{i}}\right), T_{n_{i}}\left(x_{0}\right)\right) .
$$

To estimate $\rho\left(T_{n_{i}}\left(x_{n_{i}}\right), T_{n_{i}}\left(x_{0}\right)\right)$, we use the condition (3). Then

$$
\begin{aligned}
\rho\left(T_{n_{i}}\left(x_{n_{i}}\right), T_{n_{i}}\left(x_{0}\right)\right) \leq & \alpha\left(\rho\left(x_{n_{i}}, T_{n_{i}}\left(x_{n_{i}}\right)\right)+\rho\left(x_{0}, T_{n_{i}}\left(x_{0}\right)\right)\right\} \\
& +\beta\left(\rho\left(x_{0}, T_{n_{i}}\left(x_{n_{i}}\right)\right)+\rho\left(x_{n_{i}}, T_{n_{i}}\left(x_{0}\right)\right)\right)+\gamma \rho\left(x_{n_{i}}, x_{0}\right) .
\end{aligned}
$$

For $N \leq n_{i}$, we have

$$
\rho\left(T_{n_{i}}\left(x_{n_{i}}\right), T_{n_{i}}\left(x_{0}\right)\right) \leq \alpha \rho\left(x_{0}, T_{n_{i}}\left(x_{0}\right)\right)+(\beta+\gamma) \varepsilon+\beta \rho\left(x_{n_{i}}, T_{n_{i}}\left(x_{0}\right)\right) .
$$

Hence

$$
(1-\beta) \rho\left(x_{n_{i}}, T_{n_{i}}\left(x_{0}\right)\right) \leq \alpha \rho\left(x_{0}, T_{n_{i}}\left(x_{0}\right)\right)+(\beta+\gamma) \varepsilon \text {. }
$$

Next consider $\rho\left(x_{0}, T_{n_{i}}\left(x_{0}\right)\right)$; then

$$
\rho\left(x_{0}, T_{n_{i}}\left(x_{0}\right)\right) \leq \rho\left(x_{0}, x_{n_{i}}\right)+\rho\left(x_{n_{i}}, T_{n_{i}}\left(x_{0}\right)\right) .
$$

For $N \leq n_{i}$, we have

$$
\rho\left(x_{0}, T_{n_{i}}\left(x_{0}\right)\right) \leq \varepsilon+\rho\left(x_{n_{i}}, T_{n_{i}}\left(x_{0}\right)\right) .
$$

(5) and (6) imply

$$
(1-\beta) \rho\left(x_{0}, T_{n_{i}}\left(x_{0}\right)\right) \leq(1-\beta) \varepsilon+\alpha \rho\left(x_{0},{ }^{T}{ }_{n_{i}}\left(x_{0}\right)\right)+(\beta+\gamma) \varepsilon .
$$

Hence

https://doi.org/10.1017/S0004972700041058 Published online by Cambridge University Press 


$$
\rho\left(x_{0}, T_{n_{i}}\left(x_{0}\right)\right) \leq \frac{1+\gamma}{1-\alpha-\beta} \varepsilon
$$

From (4), (5) and (7), we have

$$
\begin{aligned}
\rho\left(x_{0}, T_{0}\left(x_{0}\right)\right) & \leq 2 \varepsilon+\frac{1}{1-\beta}\left(\alpha \rho\left(x_{0}, T_{n_{i}}\left(x_{0}\right)\right)+(\beta+\gamma) \varepsilon\right) \\
& \leq\left[2+\frac{1}{1-\beta}\left[\frac{\alpha(1+\gamma)}{1-\alpha-\beta}+(\beta+\gamma)\right] \varepsilon .\right.
\end{aligned}
$$

This shows that $x_{0}$ is a fixed point of $T_{0}$. We complete the proof.

\section{References}

[1] Ljubomir B. Ćirić, "Generalized contractions and fixed-point theorems", Publ. Inst. Math. Beograd, N.S. 12 (26) (1971), 19-26.

[2] Baidyanath Ray, "Some results on fixed points and their continuity", Colzoq. Math. 27 (1973), 41-48.

[3] Ioan A. Rus, Teoria punctului fix. II Teoria punctului fix in analiza fronctională (Universitatea "Babes-Bolyai" din Cluj, Cluj, 1973).

[4] Chi Song Wong, "Conmon fixed points of two mappings", Pacific J. Math. 48 (1973), 299-312.

Department of Mathematics,

Kobe University,

Rokko, Kobe,

Japan. 